\title{
Anemometer Modified
}

\section{Kern E. Kenyon}

4632 North Lane, Del Mar, CA, USA

Correspondence to: Kern E. Kenyon, kernken@aol.com

Keywords: Anemometer, Modification

Received: January 14, 2019 Accepted: January 30, 2019 Published: February 2, 2019

Copyright $\odot 2019$ by authors and Scientific Research Publishing Inc.

This work is licensed under the Creative Commons Attribution International License (CC BY 4.0).

http://creativecommons.org/licenses/by/4.0/

\section{Open Access}

\section{ABSTRACT}

A four cup commercial anemometer is modified as follows. Two cups with arms extending from the pivot are removed. The other two half spherical cups are replaced by identical double cones, each of which is composed of two cones of different altitudes joined at the bases that are the same size. There is a stiff wire connecting the two double cones to the pivot at the midpoint so they are free to spin in the horizontal plane. They are parallel but oppositely oriented. When a fan is turned on aiming an air stream toward the double cones, they constantly rotate. Increasing the distance between the long axes of the two double cones causes the rotation rate to increase for the same setting of the fan. These results are qualitatively explained by reaction forces of a flowing fluid acting on the surfaces of solid bodies with front to back asymmetry in accord with Newton's 3rd law (action equals reaction) published by the author earlier. Besides calibrating the modified anemometer for measuring wind speed, another practical application of the concept may be the generation of electricity by the natural winds.

\section{INTRODUCTION}

Standard anemometers work very well: they rotate easily in the slightest breezes, and they work in strong winds up to about $100 \mathrm{mph}$. But is there a way to make them perform better or rotate faster for a given wind speed? From purely the perspective of fluid dynamics these anemometers have limitations. One of the two anemometers I purchased over the internet has four cups attached to 3 inch arms radiating out from a pivot. Each plastic cup is half a sphere with a half inch diameter. As each cup passes by a fixed point it has the same orientation.

Now, when a whole solid sphere is immersed and held fixed in a steady fluid flow, it is well known that eddies are shed off the back side for moderate to high mean flow speeds. Eddies produce increased friction. If the open side of a cup is trailing, there may be eddies shed behind it at almost all mean flow speeds. Of course atmospheric winds are turbulent to begin with, which is another complicating factor.

What comes to mind is to change the shape of the cup in order to try to reduce friction, and the change proposed here will at first seem rather radical. Consider a solid body composed of two cones back 
to back with a little smoothing at the joint, the surfaces of the cones are also smooth. One cone is significantly shorter in altitude than the other one, so there is front to back asymmetry. Within a steady fluid flow it does not matter which cone is trailing because the shedding of eddies will not happen or will be much reduced compared to a spherical shape, at least for moderate to slow mean speeds (for earlier work on fluids flowing by double sided cones, see [1]).

Imagine an anemometer in which the open faces of the cups are filled in with a solid longer cone pointing backwards while the front spherical face is molded into a shorter cone pointing forward. What will happen when the wind blows parallel to the long axes of the double cones? Will the system rotate or not? If so, will the sense of rotation be the same as it is before the modification?

\section{BACKGROUND}

Two identical (in weight, shape and size) double cones were crafted out of a hard wood by an experienced wood worker a few years ago (there are conceptual similarities between the earlier project and the present one as will become clear below). Maximum length of the double cones was $20 \mathrm{~cm}$ and maximum width $5 \mathrm{~cm}$, located $3.5 \mathrm{~cm}$ from one end and $16.5 \mathrm{~cm}$ from the other end. The aspect ratio of the altitudes of the two cones was based on a photograph of a fish, taken straight down from above, in a magazine or journal article [2]. Small holes were drilled along the long axes of the double cones.

Being buoyant in water the two double cones would rise up from the bottom of the deep end of a swimming pool along vertical taut plastic wires inserted through the holes. Opposite configurations of the double cones were used: the shorter cone led on one wire and the longer cone led on the other wire. Both double cones were released at depth simultaneously. Many trials runs were carried out but the results were always the same. When the blunter end led, it won the race every time by being first to break the air/water surface and by an average of about four lengths [1].

This result will startle those who have believed for a long time that the easiest way for a solid body to move through a fluid is to have the front face be pointed, and the sharper the point the better. However, there exists a qualitative physical explanation that has been fitted to the results obtained in the swimming pool involving what has been called the reaction force [2] stemming from Newton's $3^{\text {rd }}$ law of motion: action equals reaction. Flow encountering a solid body experiences accelerations and decelerations along its surfaces due to the presence and shape of the body and the fact that no flow can penetrate into the body. In particular, the body's surfaces that have a slope relative to the mean flow direction, as well as conservation of mass, are instrumental in bringing about accelerations and decelerations parallel to the solid's surfaces (the mean flow occurs far away from the solid body in any direction). It takes a force to cause an acceleration of fluid produced by the body, and then the fluid has an equal but opposite acceleration transmitted back on the body, which explains the reaction force.

If a solid body has front to back asymmetry, relative to the direction of the mean flow going by it, then a net reaction force will exist because the accelerations and decelerations on the surfaces will not be equal and therefore will not cancel each other out. In the case of the double cone the magnitudes of the accelerations and decelerations are greater on the surface of the cone with the smaller altitude. Consequently, the net reaction force will always point outward away from the shorter cone whether it is heading into the mean flow or the other way around.

Another way to put it is: why the accelerations and decelerations are greater on the surface of the shorter cone is explained by the observed fact, going back to Leonardo da Vinci watching rivers passing by rocks, that the greatest speed of flow tangent to the solid body occurs where the body is thickest. From the mean flow to the thickest part of the double cone is a smaller distance along the shorter cone, so a given difference is speed of flow over a shorter distance produces a greater acceleration (or deceleration depending on which way the flow is directed).

\section{PROOF OF CONCEPT}

Go back to the anemometer for answers to the questions at the end of Section 1. Connect together the 
two wooden double cones discussed above by a rigid but bendable wire sitting at its mid-point on a vertical pivot, taken from the anemometer, so that they can rotate freely in the horizontal plane. In the first trial the separation between the long axes of the double cones was 6.5 inches and they were anti-parallel, meaning that the shorter cones pointed in opposite directions. Then the fan was turned on blowing an air stream horizontally toward the double cones. They rotated rather slowly but constantly, and a 30 second video was made. The sense of rotation was the same as if the open faces of the half spherical cups had been filled in by the longer altitude cones pointing backward and the spherical caps had been replaced by the shorter cones. And there were only two double cones instead of four cups.

A guess was made that if the separation between the long axes of the double cones had been larger, the rotation rate would also have been larger for the same setting of the fan. This conjecture was verified in the second trial, and another 30 second video was made. From a 6.5 inch to an 11 inch separation the increase in rotation rate was obvious from comparing the two videos.

Taken together these two results are consistent with the idea that for a given reaction force the rotation rate will increase with increasing radius from the pivot because the torque in the system is thereby increased.

\section{DISCUSSION}

Various parameters of the problem at hand can be changed depending either on the intended practical use or for academic reasons. For example, if it is desired to calibrate the modified anemometer to measure wind speeds, then solid bodies of rather expensive hard woods would not be needed. That was chosen for the buoyancy effect in the early trials in water. Also more than two double cones could be tried. Should it occur that the concept might be adapted for generating electricity, which suggestion is not too farfetched of a notion, then the whole scale of the apparatus could be made a lot bigger.

From the scientific point of view, the results so far are real but few and qualitative. There is plenty of room for more work including quantitative relationships. Along with videos that are hard to publish, graphs of variables plotted against one another are needed.

One step toward being more quantitative has already occurred by calculating the reaction force on a circular arc wing and finding out under what conditions its magnitude can equal that of the lift force on the same wing [3]. It turns out that the order of magnitudes of the two different forces is the same if the maximum thickness of the wing is not too small. Of course the lift forces on the double cones cancel out because of the circular symmetry about the long axes.

\section{CONCLUSION}

Why anemometer cups have the shape they do is a puzzle because fluid dynamics allows other possibilities that may work just as well or even perhaps better. One new arrangement is proposed here involving double sided cones and preliminary trials with them show promise. Also applications of the modified anemometer may not be limited to just measuring the speed of winds as pointed out above.

\section{ACKNOWLEDGEMENTS}

Comments by the reviewer and Tony Sturges helped improve the explanations.

\section{CONFLICTS OF INTEREST}

The author declares no conflicts of interest regarding the publication of this paper.

\section{REFERENCES}

1. Kenyon, K.E. (2017) Asymmetric Solids Move Faster in Water When the Blunter End Leads. European Journal of Science and Theology, 6, 61-64. 
2. Kenyon, K.E. (2006) Reaction Forces of Fluids on Solid Boundaries. Physics Essays, 19, 507-516. https://doi.org/10.4006/1.3028856

3. Kenyon, K.E. (2018) Fluid Reaction Force on Solids. Natural Science, 10, 416-419. https://doi.org/10.4236/ns.2018.1010039 